\title{
Single-grating interferometer for high-resolution phase-contrast imaging at synchrotron radiation sources
}

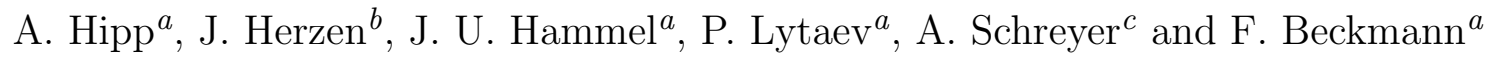 \\ ${ }^{a}$ Helmholtz-Zentrum Geesthacht, Max-Planck-Strasse 1, Geesthacht, Germany; \\ ${ }^{b}$ Technische Universität München, James-Frank Strasse 1, Garching, Germany; \\ ${ }^{c}$ European Spallation Source (ESS), Tunavågen 24, Lund, Sweden
}

\begin{abstract}
Synchrotron X-ray imaging is constantly achieving higher spatial resolution. In the field of grating-based phasecontrast imaging, these developments allow to directly resolve the interference patterns created by a phase grating without need for a analyzer grating. In this study we analyzed the performance of a single-grating interferometer and compared it to a conventional double-grating interferometer. Based on simulations and measurements of a test phantom we evaluated the sensitivity, resolution and signal to noise ratios of different setup configurations.
\end{abstract}

Keywords: Phase Contrast, Grating Interferometer, X-ray Imaging, Synchrotron Radiation, Microtomography

\section{INTRODUCTION}

Differential phase-contrast imaging (DPC) has proven to be a valuable tool when investigating weak absorbing materials like soft tissue, due to its increased contrast compared to conventional absorption-contrast imaging. ${ }^{1-3}$ The most common type of grating interferometer consists of two gratings, a phase grating and an analyzer grating. ${ }^{3,4}$ Although a two-grating interferometer comes with a high sensitivity its performance is mainly limited by the feasible aspect ratio of the analyzer-grating structures. The period of these structures (state of the art $2.4 \mu \mathrm{m}$ ) has to be smaller than the used pixel size and have to be highly absorbing which strongly limits the usable energy range. To achieve grating structures with the necessary aspect ratio is still the critical part in grating production. ${ }^{5,6}$ In contrast to this, a single-grating interferometer comes with several advantages: The absence of an analyzer grating increases the photon flux at the detector plane by almost a factor of two and at the same time allows for using this type of interferometer at any energy. Additionally, the setup itself is more stable and easily adjustable. With a single-grating interferometer it is also possible to use two different modes of phase-retrieval: The so-called stepping approach ${ }^{7}$ and the singe-shot or fringe analysis approach. ${ }^{8,9}$ These two modes make the same interferometer usable for high-resolution phase-contrast imaging and for very fast measurements. Main requirement for this type of setup is a detector system with a high spatial resolution, that allows to resolve the interference pattern directly. The microtomography endstation at the beamline $\mathrm{P} 05^{10,11}$ is operated by the Helmholtz-Zentrum Geesthacht at the synchrotron radiation storage ring PETRA 3 at DESY, Hamburg, Germany. The imaging detector consists of a CCD-camera and a magnifying optics, which results in an effective pixel size between $0.3 \mu \mathrm{m}$ and $2.4 \mu \mathrm{m}$. This is an ideal basis for high-resolution phase-contrast imaging using a single-grating interferometer. Here we will present our investigations of a single-grating interferometer based on simulations. Focus of these calculations is on the reachable sensitivity, depending on the grating period and the effective pixel size, as well as on the spatial resolution of the setup. For experimental verification we will compare the performance from a conventional 2-grating setup to the single-grating interferometer on the basis of a test phantom tomography.

Further author information:

A. Hipp: E-mail: alexander.hipp@hzg.de, Telephone: +49 (0)40 89985301

Developments in X-Ray Tomography X, edited by Stuart R. Stock, Bert Müller, Ge Wang, Proc. of SPIE Vol. 9967, 996718 - (c) 2016 SPIE · CCC code: 0277-786X/16/\$18 · doi: 10.1117/12.2237582 


\section{SIMULATION METHOD}

For simulations in this study all gratings were assumed to be perfectly fabricated and therefore were represented by one-dimensional functions with a duty cycle (ratio of bar width to grating period) of 0.5. The incoming wave was always assumed as perfect plane wave. For a certain phase shift $\Delta \phi$ and the attenuation coefficient $\mu$, dependent on the grating material and X-ray energy, the resulting wave front behind the phase grating with height $d_{0}$ is defined as:

$$
A_{0}(x)=e^{-\mu d(x)} e^{-i \Delta \phi(x)}
$$

The dependency on $\mathrm{x}$ of the variables is used to distinguish between grating bar $\left(d=d_{0}\right)$ and gap $(d=0)$. This modified wave front is propagated along the z-axis applying the Fresnel propagator

$$
\widetilde{P}_{z}\left(k_{x}\right)=e^{i k z} e^{i k k_{x}^{2} / 2 k}
$$

in Fourier space with $\mathcal{F}$ denoting a Fourier transformation and $\mathcal{F}^{1}$ denoting an inverse Fourier transformation:

$$
A_{z}(x)=\mathcal{F}^{-1}\left\{\mathcal{F}\left\{A_{0}(x)\right\} \widetilde{P}_{z}(x)\right\} .
$$

The resulting intensity pattern at the detector plane is then given as

$$
I_{Z}(x)=\left|A_{z}(x)\right|^{2} .
$$

These equations were used to simulate a single grating interferometer with varying grating period $p$ and adjusted pixels size $s\left(p_{1}=10 \mu \mathrm{m} / s_{1}=2.4 \mu \mathrm{m}, p_{2}=7 \mu \mathrm{m} / s_{2}=1.2 \mu \mathrm{m}, p_{3}=5 \mu \mathrm{m} / \mathrm{s}_{3}=1.2 \mu \mathrm{m}\right.$ and $p_{4}=2.4 \mu \mathrm{m} /$ $\left.s_{4}=0.6 \mu \mathrm{m}\right)$ for different distances. To analyze the performance of each setup the projection of a test pattern, consisting of silicon rods with a square cross-section of different edge-lengths (Figure 1), was simulated using the stepping approach. For later comparison with the experimental data, the simulations assumed a photon energy of $20 \mathrm{keV}$.

\section{EXPERIMENTAL SETUP}

As sample for this study we prepared a test phantom consisting of several polymer and metal rods embedded in hot-melt glue (composition not known). The used material are Polyamide 6.6 (PA66, Ø 1.5mm), Polymethylmethacrylate (PMMA, Ø $1.1 \mathrm{~mm}$ ), Magnesium $(\mathrm{Mg}, \varnothing 1.5 \mathrm{~mm}$ ), Polychlortrifluorethylene (PCTFE, $\varnothing 1.5 \mathrm{~mm}$ ), Polytetrafluorethylene (PTFE, Ø $1 \mathrm{~mm}$ ), Aluminum (Al, $\varnothing 0.25 \mathrm{~mm}$ ).

The CCD camera used for this experiment is based on a Kodak KAF-09000 chip with 3056 pixels in width and a pixel size of $12 \times 12 \mu \mathrm{m}^{2}$ and a depth of 16-bit. Our phase grating had a period of $10 \mu \mathrm{m}$ with a structure height of $21 \mu \mathrm{m}$ made of nickel for the single-grating setup and a period of $4.8 \mu \mathrm{m}$ with a structure height of $14 \mu \mathrm{m}$ for the two-grating setup. The analyzer grating needed for the two-grating setup had a period of $2.4 \mu \mathrm{m}$ and gold structures with a height of roughly $100 \mu \mathrm{m}$. All these gratings were produced by the Institute for Microstructure Technology at the Karlsruhe Institute of Technology using the LIGA-process. ${ }^{12}$ The experiment was performed at an photon energy of $20 \mathrm{keV}$. At this energy the gratings induce at phase shift of $3 \pi / 2$ for the single-grating setup and a $\pi$-shift for the two-grating setup. With the single grating setup, the detector was positioned $40 \mathrm{~cm}$ from the phase grating, which corresponds to half of the first fractional Talbot distance, to avoid too strong blurring occurring from a strong signal and the beamplitter characteristics. For the two-grating setup the tomogram was taken at the third fractional Talbot distance $(13.9 \mathrm{~cm})$. For each scan 900 projections were taken covering a rotation of $180^{\circ}$, with each projection consisting of a stepping series with three steps (four images in total). The retrieved phase projections were binned by a factor of two in case of the single grating setup where we need to resolve the interference pattern. For the data obtained with the two grating setup, the binning was already performed before phase retrieval. The data-sets were reconstructed using filtered back projection $^{13}$ retrieving volumes with an isotropic voxel size of $(4.94 \mu \mathrm{m})^{3}$. 

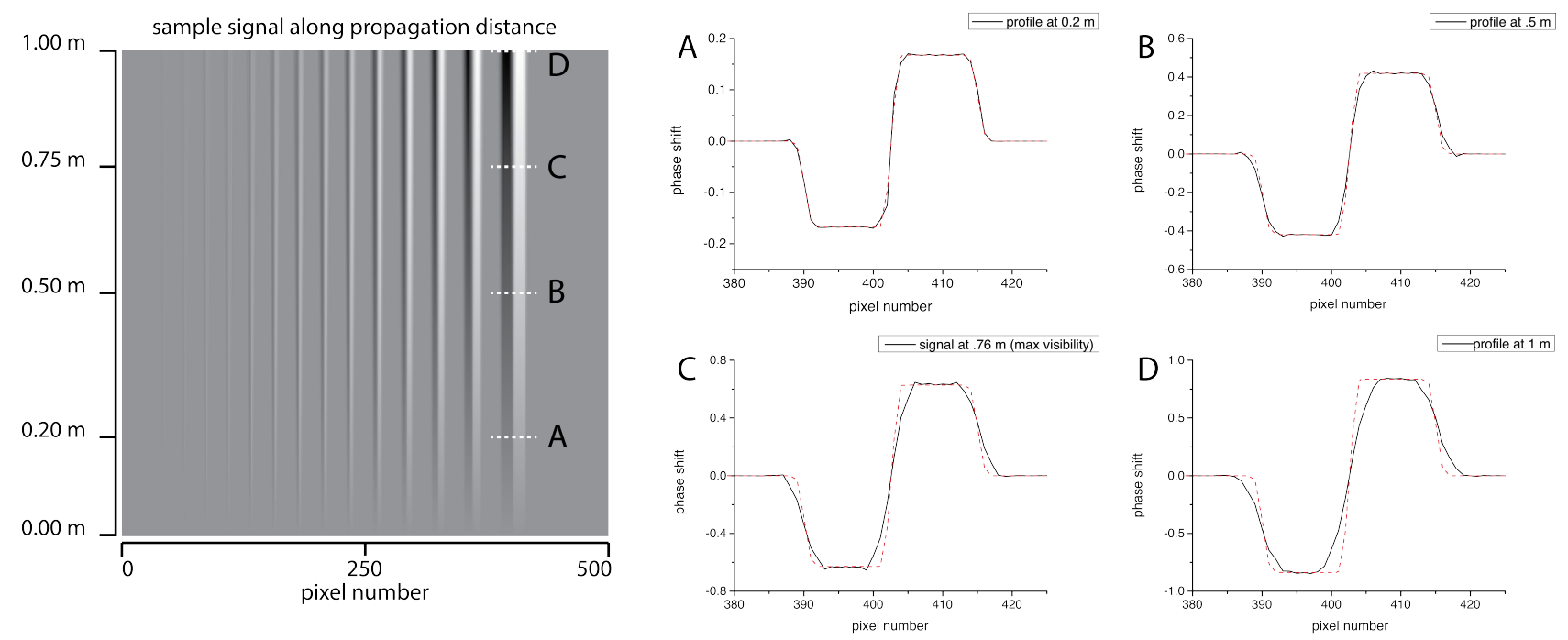

Figure 1. The image shows the simulated projected profile of the test sample along the propagation distance. The test sample consists of several silicon rods with a squared cross-section sized between $1 \mu \mathrm{m}$ and $60 \mu \mathrm{m}$. The phase-projection was retrieved from a stepping-scan with 5 steps and with a grating period of $10 \mu \mathrm{m}$. Structures below $5 \mu \mathrm{m}$ are detectable. The plotted profiles of the largest rod at different distances (A, B, C, D) show significant blurring of the edges already at the 1st fractional Talbot distance $(\mathrm{C})$. At short distances $(\mathrm{A}, \mathrm{B})$ the projected profile is in very good agreement with the expected signal (red dashed curve).

\section{RESULTS}

\subsection{Simulations}

The simulations for every grating interferometer show significant blurring at the edges of the test patter, increasing with the propagation distance (Figure 1). This blurring is a result of the beamsplitter characteristic of the phase grating and can be observed due to the small pixel sizes used. From the diffraction equation for small angles

$$
\theta_{m}=\arcsin (m \lambda / p) \approx m \lambda / p
$$

we see that the blurring will be inversely proportional to the grating period $\mathrm{p}$ and is scaled proportional to the propagation distance due to the geometric magnification. Figure 2 shows how the blurring affects the obtained phase-shift values at the edge of a rod. The red dotted lines mark the area, where the deviation of the signal from the theoretical value is lower than $5 \%$. The blue lines mark the odd fractional Talbot-distances. The distinct distance where the signal rises over $105 \%$ or falls below $95 \%$ of the theoretical signal will be called critical distance $d_{c}$ in the following. The Talbot-distance is inversely proportional to $p^{2}$, thus a larger relative propagation distance (in terms of the Talbot-distance) is applicable the smaller the grating period is. To compare the performance of the different setups we calculated the sensitivities of each setup, which depends on the propagation distance $d$, grating period $p$, visibility $V$ and the photon counts $N$.

$$
S \sim d / p \cdot V \sqrt{N}
$$

Figure 3 shows the comparison of the sensitivity for the case that the visibility of each setup is almost not affected by the optical components of the whole setup and a more realistic case where a Gaussian-shaped PSF $(\mathrm{FWHM}=1 \mu \mathrm{m})$ is added to the simulation. The comparison of the sensitivity up to the relative critical distance shows an advantage in reducing the grating period and enlarging the relative propagation distance, if the visibility is comparable to a setup with a larger period. For a camera system showing a not negligible PSF the sensitivity drops rapidly with smaller grating periods, because the visibility is lowered until the interference pattern is not resolvable anymore. In this case such small grating periods can only be used in a two-grating setup. For this type 

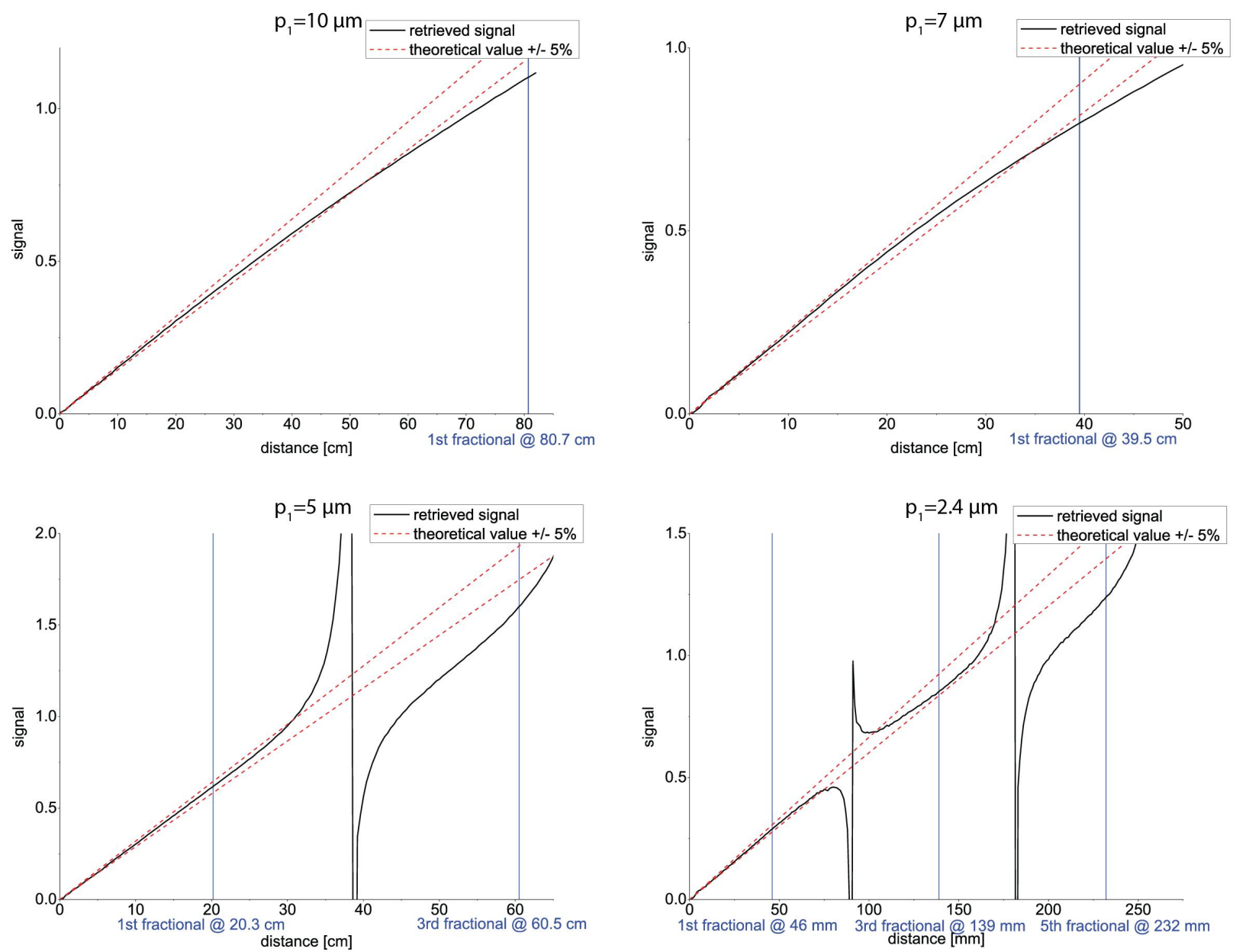

Figure 2. The image shows the obtained signal of single-grating setups with varying phase-grating periods. The red dashed lines mark the area where the signal varies less than $5 \%$ from the theoretical value. For the setups with a $10 \mu m$ and $7 \mu \mathrm{m}$ period the critical distance where the signal varies more than $5 \%$ is shorter than the 1st fractional Talbot-distance. For the setups with a $5 \mu \mathrm{m}$ and $2.4 \mu \mathrm{m}$ periods the exact position of the critical distance is covered by imprecise signal values in the area of the 2 nd and 4 th fractional Talbot-distance.

of setup the second grating preserves the contrast of the interference pattern, but reduces the photon counts to almost $50 \%$. Figure 4 shows the projection of the test pattern at the distance with maximal sensitivity below the critical distance for a setup with a period of $10 \mu \mathrm{m}$ and $2.4 \mu \mathrm{m}$, respectively. For both setups the smallest structures with $1 \mu \mathrm{m}$ width are still detectable. Nevertheless, the achieved signal for the small structures strongly differs from the theoretical value up to a structure size of $12 \mu \mathrm{m}$ and $9 \mu \mathrm{m}$, respectively. By reducing the propagation distance of the $2.4 \mu \mathrm{m}$ period setup to the first fractional Talbot-distance, correct values are achieved for structures with a size of $3 \mu m$ or larger.

\subsection{Experimental}

To compare the performance of the single-grating setup with a conventional two-grating setup, we analysed the reconstructed volume of a test-phantom as described in section 3. As main performance indicator the quantitative values obtained from the different phantom materials were taken together with the achieved signal to noise ratio. The obtained values are listen in table 1 . The comparison of the results shows, that both setups 

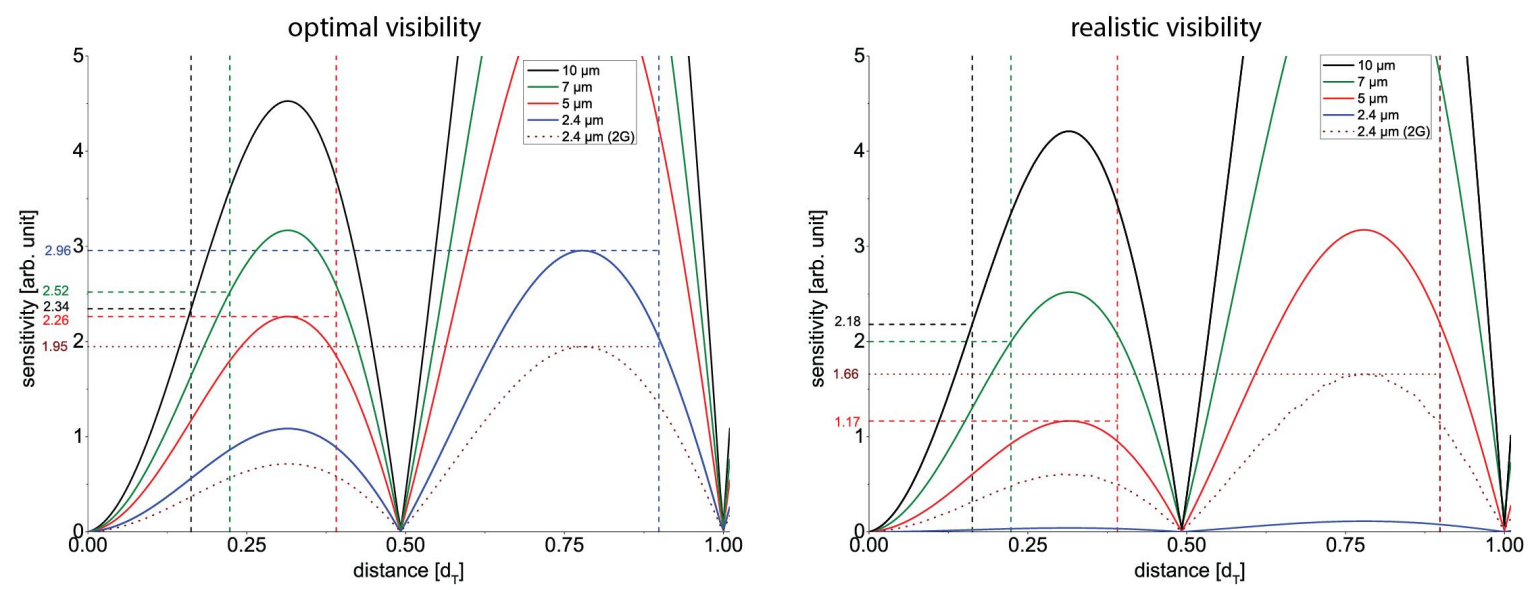

Figure 3. The comparison of the sensitivity shows an advantage in using smaller grating periods when the achieved visibility is in the same range as for larger grating periods (optimal visibility). By adding a not negligible point spread function to the simulations (realistic visibility), the sensitivity drops dramatically for small grating periods. The plots are compared to a conventional two-grating setup with $2.4 \mu \mathrm{m}$ grating periods.
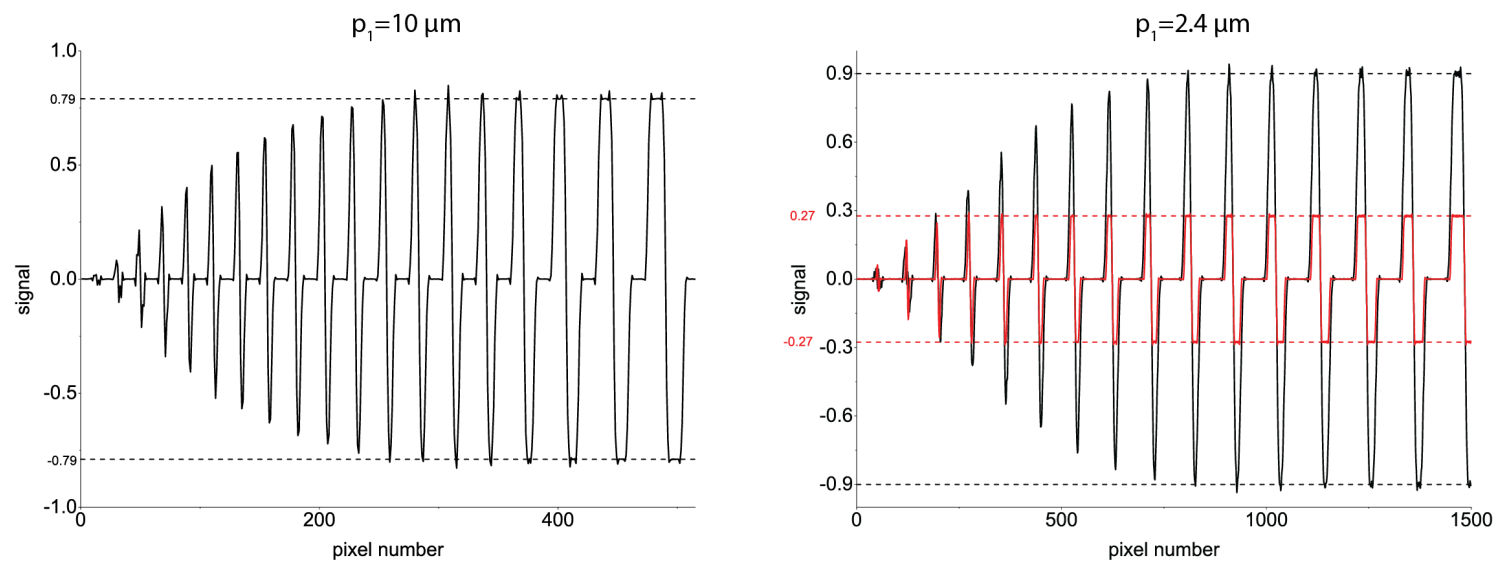

Figure 4. One dimensional projection of a test pattern for a setup with a $10 \mu \mathrm{m}$ period grating and a $2.4 \mu \mathrm{m}$ period grating at the distance for maximal sensitivity ( $\tilde{4} 0 \mathrm{~cm}$ for single grating, 3 rd fractional for double-grating). In both setups, structures down to a size of $1 \mu \mathrm{m}$ are visible, but lack of a correct signal value for the small structures. For a shorter propagation distance (1st fractional Talbot-distance) the $2.4 \mu \mathrm{m}$ period setup is able to give a correct (but much smaller) signal for structures to a size down to $3 \mu \mathrm{m}$ (red lines). For the single grating setup, the visibility would drop too fast for even smaller distances which doesn't allow for reasonable measurement.

reach a comparable precission in the retrieved values. The signal to noise ratio on the other hand is clearly stronger for the single-grating setup. Main reason for this lies in the absence of the analyzer-grating. Without this second grating the photon flux at the detector plate is almost doubled. An additional effect is that grating imperfections of the analyser grating are imprinted to the single projections and therefore cause artifacts in the reconstructed slices. Due to the small grating periods of the two-grating setup, it is more vulnerable to phase-wrapping than the single-grating setup. Therefore more artifacts at the edges of the single rods occur. These streak artifacts also lower the signal to noise ratio and are clearly visible in the reconstruction shown in figure 5 . 
Table 1. Quantitative values retrieved from the tomographic reconstruction using the single-grating and the double-grating setup. The $\delta$-values are given to compare the precission of the data. The signal to noise ratio (SNR) can be used to compare the image quality.

\begin{tabular}{lc|cc|cc}
\hline \multirow{2}{*}{ Material } & \multirow{2}{*}{ Literature } & \multicolumn{2}{|c|}{ Single-Grating } & \multicolumn{2}{c}{ Double-Grating } \\
& & $\delta\left[10^{-6}\right]$ & SNR & $\delta\left[10^{-6}\right]$ & 6.33 \\
PAN6 & 6.46 & 6.60 & 115 & 6.74 & 62 \\
PMMA & 6.67 & 6.75 & 114 & 8.60 & 45 \\
Mg & 8.95 & 8.90 & 106 & 10.3 & 78 \\
PCTFE & 10.7 & 10.3 & 144 & 10.4 & 147 \\
PTFE & 11.0 & 10.8 & 239 & 12.2 & 113 \\
Al & 13.6 & 12.9 & 244 & & \\
\hline
\end{tabular}
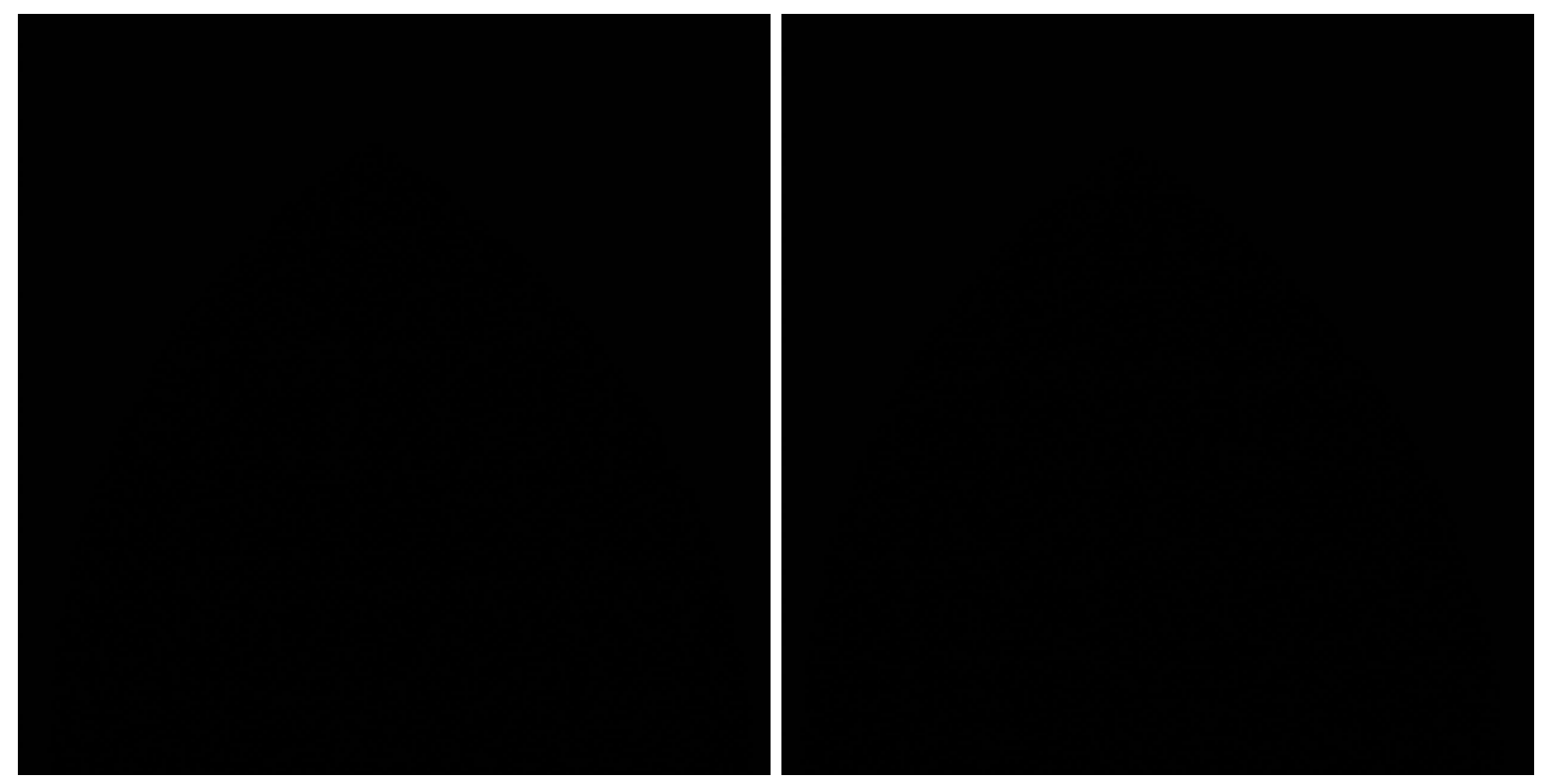

Figure 5. Reconstructed slices of the test phantom. The reconstruction obtained with the two-grating setup (left) shows significantly more streak artifacts than the one obtained with the single-grating setup (right).

\section{SUMMARY AND DISCUSSION}

The simulations of a single-grating interferometer predict the possibility to realize a high sensitive setup for differential phase-contrast measurements, which is limited by the available propagation distance. Due to the beam-splitter characteristic of the phase-grating and the small pixel sizes the usable propagation distance is severely limited to avoid strong blurring of the projection. Smaller grating periods allow to go for higher fractions of the Talbot distance, but are more vulnerable to the point spread function of the optical components of the experimental setup. The investigation of the reachable resolution of the different single grating setups reveal that even with a period of the phase grating structures of $10 \mu \mathrm{m}$ sample structures down to $1 \mu \mathrm{m}$ are detectable. Nevertheless, all setups fail giving reliable signal values for structures in the range of $1 \mu \mathrm{m}$ and $10 \mu \mathrm{m}$ at distances for high sensitivity. Aiming at a high-resolution single-grating interferometer these limitations lead to a setup with a small phase-grating period (state of the art: $2.4 \mu \mathrm{m}$ ). Using such small grating periods require a detector system of the experiment where the FWHM of the PSF does not exceed 10\% of the grating period to achieve an adequate visibility. For an experimental setup with a larger PSF, a two-grating setup is required using these periods. Additionally, the propagation distance between grating and detector has to be kept as short as possible to avoid loss of accuracy due to image blurring occurring from the beam-splitter characteristic of the phase grating and the signal itself. The experimental evaluation of the tested single-grating setup and the 
comparison to a two-grating setup confirm the results from the simulations. In our measurements we could show the signal to noise ratio and image quality is much higher for the single-grating setup than for the two-grating setup. Especially for grating interferometers aiming on a high spatial resolution the setup benefits a lot from the absence of an analyzer grating. Due to the pixel size in the range of the grating period, a two-grating interferometer shows a strong vulnerability to grating defects. A single-grating interferometer not only profits from the easy setup and stability, but also from the large propagation distance which allows for a very high sensitivity. To analyse the performance in terms of spatial resolution further experiments have to be performed. Especially the influence of the large propagation distance in connection with materials giving a strong signal is a critical point that has to be taken care of.

\section{ACKNOWLEDGMENTS}

First of all our gratitude goes to the Institute of Microstructure Technology of the Karlsruhe Institute of Technology, a partner in the Virtual Institute: 'New X-ray analytic methods in material science', for supply of all gratings necessary for this study. We would like to thank our first users from the Biomaterials Science Center of the University Basel for testing our new setup on biomedical applications.

The work was funded by the Helmholtz-Portfolio Theme Technology and Medicine.

\section{REFERENCES}

1. R. Fitzgerald, "Phase-Sensitive X-Ray Imaging," Physics Today 53, p. 23, July 2000.

2. F. Beckmann, U. Bonse, F. Busch, and O. Günnewig, "X-ray microtomography (microCT) using phase contrast for the investigation of organic matter.," Journal of computer assisted tomography 21(4), pp. 539-53, 1997.

3. A. Momose, "Phase-sensitive imaging and phase tomography using X-ray interferometers," Optics Express 11, p. 2303, Sept. 2003.

4. C. David, B. Nohammer, H. H. Solak, and E. Ziegler, "Differential x-ray phase contrast imaging using a shearing interferometer," Applied Physics Letters 81(17), p. 3287, 2002.

5. E. Reznikova, J. Mohr, M. Boerner, V. Nazmov, and P.-J. Jakobs, "Soft X-ray lithography of high aspect ratio SU8 submicron structures," Microsystem Technologies 14, pp. 1683-1688, Jan. 2008.

6. J. Mohr, T. Grund, D. Kunka, J. Kenntner, J. Leuthold, J. Meiser, J. Schulz, and M. Walter, "High aspect ratio gratings for X-ray phase contrast imaging," in AIP Conference Proceedings, pp. 41-50, 2012.

7. T. Weitkamp, A. Diaz, C. David, F. Pfeiffer, M. Stampanoni, P. Cloetens, and E. Ziegler, "X-ray phase imaging with a grating interferometer.," Optics Express 13, pp. 6296-304, Aug. 2005.

8. M. Takeda, H. Ina, and S. Kobayashi, "Fourier-transform method of fringe-pattern analysis for computerbased topography and interferometry," Journal of the Optical Society of America 72, p. 156, Jan. 1982.

9. E. E. Bennett, R. Kopace, A. F. Stein, and H. Wen, "A grating-based single-shot X-ray phase contrast and diffraction method for in vivo imaging," Medical Physics 37(11), p. 6047, 2010.

10. I. Greving, F. Wilde, M. Ogurreck, J. Herzen, J. U. Hammel, A. Hipp, F. Friedrich, L. Lottermoser, T. Dose, H. Burmester, M. Müller, and F. Beckmann, "P05: Imaging beamline at PETRA III," in Proceedings of SPIE, 2014.

11. A. Haibel, F. Beckmann, T. Dose, J. Herzen, M. Ogurreck, M. Müller, and A. Schreyer, "Latest developments in microtomography and nanotomography at PETRA III," Powder Diffraction 25, pp. 161-164, Feb. 2012.

12. W. Bacher, W. Menz, and J. Mohr, "The LIGA technique and its potential for microsystems-a survey," IEEE Transactions on Industrial Electronics 42(5), pp. 431-441, 1995.

13. F. Pfeiffer, O. Bunk, C. Kottler, and C. David, "Tomographic reconstruction of three-dimensional objects from hard X-ray differential phase contrast projection images," Nuclear Instruments and Methods in Physics Research Section A: Accelerators, Spectrometers, Detectors and Associated Equipment 580, pp. 925-928, Oct. 2007. 